Editorial

\title{
Cancer Nano-Immunoengineering: The Marriage of Immunoengineering and Nanotechnology for Cancer Therapy
}

\author{
Weilin Jin, Daxiang Cui \\ Institute of Nano Biomedicine and Engineering, Key Laboratory of Thin Film and Microfabrication Technology of Ministry of \\ Education, Department of Instrument Science and Engineering, School of Electronic Information and Electrical Engineering, \\ Shanghai Jiao Tong University, 800 Dongchuan Road, Shanghai 200240, China.
}

Corresponding author. E-mail: dxcui@sjtu.edu.cn

Published: Jun. 29, 2016.

Citation: Weilin Jin, Daxiang Cui, Cancer Nano-Immunoengineering: The Marriage of Immunoengineering and Nanotechnology for Cancer Therapy. Nano Biomed. Eng., 2016, 8(2): 105-107.

DOI: 10.5101/nbe.v8i2.p105-107.

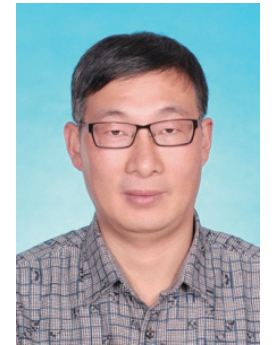

Weilin Jin obtained his doctorate of medicine (M.D, 2004) in Neurobiology from the Fourth Military Medical University, China. He is currently an Associate Professor in Shanghai Jiao Tong University. His research interests include the fundamental issue of cancer epigenetics, molecular targeted therapy and immunotherapy in brain tumor.

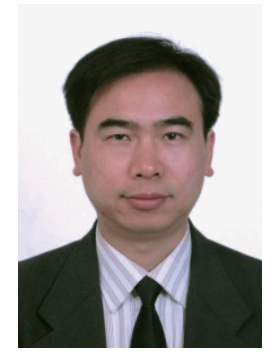

Daxiang Cui is Professor at the Institute of Nano Biomedicine and Engineering, Shanghai JiaoTong University, and Editorin-chief of Nano Biomediicne and Engineering. His research interests focus on nano theranostic technologies and their clinical translations.
Cancer is a major public health problem in the world. There is still a long way to go before we can manage cancer as a chronic disease or cure cancer. And now, cancer immunotherapy, a strategy that harnesses the human immune system to fight against cancer is paving the path to curing it.

Activating the immune system for therapeutic benefit in cancer has become a goal in immunology and oncology. Coley's toxin (a mixture of heatkilled bacteria) has been recognized as the first cancer immunotherapy. Since then various cancer immunotherapeutic methods have been explored and evaluated. After decades of disappointment, the tide has finally changed due to the success of recent proofof-concept clinical trials. To date, at least two different techniques show signs of helping some cancer patients. One involves immune-checkpoint blockade antibodies (e.g. by antibodies that block PD-1, PD-L1, or CTLA4) that release a brake on $\mathrm{T}$ cells, giving them the power to capture tumors. The other involves genetically modifying an individual $>\mathrm{s} T$ cells outside the body (e.g. tumor antigen-specific TCRs or chimeric antigenreceptor (CAR) gene-transduced blood $\mathrm{T}$ cells) to enable them to target cancer, and then reinfusing them, so that they can kill cancer cells [1]. Hence, cancer 
immunotherapy was selected as Breakthrough of the Year for 2013 by Science [3].

Although cancer immunotherapy can lead to durable outcomes, the percentage of patients who respond to this disruptive approach remains modest to date. Based on various studies on mouse tumor models, immunotherapy based on cancer-specific $\mathrm{T}$ cells has been considered as an attractive therapeutic strategy. However, most of the cancer vaccines using tumor antigens, dendritic cells or modified cancer cells, which are supposed to induce anti-tumor $\mathrm{T}$ cells to eradicate cancer cells, have failed to show significant antitumor effects in phase III trials [2].

A central question in cancer immunology is whether recognition of tumor antigens by the immune system leads to activation (i.e., surveillance) or tolerance. The effect of traditional immunotherapy is modest, frequently due to tumor escape and resistance of multiple mechanisms [3]. Advances in protein engineering and materials science have contributed to novel nanoscale targeting approaches that may bring new hope to cancer patients. For example, human CIK cells labeled with gold nanorods were used for photothermal-immunological combined therapy of in vivo gastric cancer models, and maximal tumor growth inhibition was achieved. The fact highly suggests that available nanotechnology can be used for enhanced immunotherapeutic efficacy [4]. Cancer nanotechnology or cancer nanomedicine has provided a practical solution for overcoming the limitations of traditional immunotherapy.

Nanotechnology has the potential to revolutionize cancer theranostics. Encouragingly, some nanostructures have already been demonstrated to be applicable as a drug delivery system, and the application of nanotechnology can improve the efficacy of immunostimulatory small molecules by altering their co-localization, bio-distribution and release kinetics [5]-[7]. Extensive evidence demonstrates that nanocarriers (including micelles, liposomes, polymer-drug conjugates, metal nanoparticles, solid lipid nanoparticles and biodegradable nanoparticles) could be used for the cellular transfer of immune effectors for active and passive nanoimmunotherapy [3]. Nanocarriers can be used to enhance cancer immunotherapy at four alternative pathways: (1) deliver cancer vaccine antigens and adjuvants to dendritic cells, (2) stimulate $\mathrm{T}$ cells directly as artificial antigen presenting cells,
(3) concentrate immunostimulatory compounds in the immunosuppressive tumor microenvironment, and (4) deliver supportive drugs to $\mathrm{T}$ cells in the circulation [2]. The smart engineering of nanocarriers based on the unique features of the tumor microenvironment and extra-/intra-cellular conditions of tumor cells can greatly tip the triangle immunobalance among host, tumor and nanoparticles in favor of antitumor responses, which shows a promising prospect for nanoimmunotherapy [3].

Emerging evidence confirms that cancer immunotherapies have started to combine with nanotechnology and thus created a novel area, that is cancer nano-immunoengineering. Cancer nanoimmunoengineering can generate adaptive and durable responses, yielding much more robust antitumor effects when they are formulated in nanoparticles or scaffolds than when they are administered as free drugs. Cancer nano-immunoengineering aims to fully use the advantages of nanoparticles and nano-effects, enhance immunotherapeutic cells' killing tumor effects, regulate immuno-network, and realize precise cancer targeted theranostics. How to promote the development of nano-immunoengineeirng and how to clarify the mechanism of interaction between immunonetworks and nanoparticles have become novel key scientific problems.

Cancer nano-immunoengineering is a promising area worthy of further consideration and deep investigation. A series of products based on cancer nano-immunoengineering will affect clinical cancer theranostics in near future.

\section{References}

[1] J. Couzin-Frankel, Breakthrough of the year 2013. Cancer immunotherapy. Science, 2013, 342: 1432-1433.

[2] M.S. Goldberg, Immunoengineering: how nanotechnology can enhance cancer immunotherapy. Cell, 2015,161(2): 201-204.

[3] W. Li, H. Wei, H. Li, et al., Cancer nanoimmunotherapy using advanced pharmaceutical nanotechnology. Nanomedicine (Lond), 2014 , 9(16): 2587-2605.

[4] Y. Yang, J. Zhang, F. Xia, et al., Human CIK cells loaded with Au nanorods as a theranostic platform for targeted photoacoustic imaging and enhanced immunotherapy and photothermal therapy. Nanoscale Res. Lett., 2016, 11: 285.

[5] C. Li, S. Liang, C. Zhang, et al., Allogenic dendritic cell and tumor cell fused vaccine for targeted imaging and enhanced immunotherapeuticefficacy of gastric cancer. Biomaterials, 2015, 54: 177-187.

[6] D. Cui, Y. Yang, J. Zhang, et al., Gold nanoprims as theranostic platform for enhanced drug delivery, targeted imaging, and photothermal therapy. Nanomedicine, 2016, 
12: 456.

[7] D. Cui, C. Zhang, B. Liu, et al., Regression of gastric cancer by systemic injection of RNA nanoparticles carrying both ligand and siRNA. Sci. Rep., 2015: 10726.
Copyright $₫ 2016$ Weilin Jin, Daxiang Cui. This is an openaccess article distributed under the terms of the Creative Commons Attribution License, which permits unrestricted use, distribution, and reproduction in any medium, provided the original author and source are credited. 